\title{
Is asthma associated with COVID-19 infection? A UK Biobank analysis
}

\author{
Caroline J. Lodge $\mathbb{Q}^{1,6}$, Alice Doherty ${ }^{1,6}$, Dinh S. Bui ${ }^{1}$, Raisa Cassim $\mathbb{\mathbb { D }}^{1}$, Adrian J. Lowe $\mathbb{\mathbb { D }}^{1}$, \\ Alvar Agusti ${ }^{2,3,4,5}$, Melissa A. Russell $\mathbb{1}^{1,6}$ and Shyamali C. Dharmage $\mathbb{1}^{1,6}$
}

${ }^{1}$ Allergy and Lung Health Unit, Centre of Epidemiology and Biostatistics, Melbourne School of Population and Global Health, the University of Melbourne, Melbourne, VIC, Australia. ${ }^{2}$ Centro de Investigation Biomédica en Red Enfermedades Respiratorias (CIBERES), Spain. ${ }^{3}$ Institut d'Investigacions Biomediques August Pi i Sunyer (IDIBAPS), Barcelona, Spain. ${ }^{4}$ Respiratory Institute, Hospital Clinic, Barcelona, Spain. ${ }^{5}$ University of Barcelona, Spain. ${ }^{6}$ Equal first/senior.

Corresponding author: Caroline J. Lodge (clodge@unimelb.edu.au)

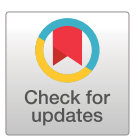

This version is distributed under the terms of the Creative Commons Attribution NonCommercial Licence 4.0. For commercial reproduction rights and permissions contact permissions@ersnet.org

This article has supplementary material available from openres. ersjournals.com

Received: 4 May 2021 Accepted: 20 Aug 2021

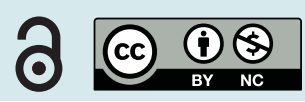

Shareable abstract (@ERSpublications)

Reduced risk of positive \#SARSCoV2 test was associated with early-onset asthma in males, nonsmokers, overweight/obese and non-black participants. Lack of phenotyping and unappreciated interactions may contribute to inconsistent findings for asthma and \#COVID19. https://bit.ly/3yn3Nvi

Cite this article as: Lodge CJ, Doherty A, Bui DS, et al. Is asthma associated with COVID-19 infection? A UK Biobank analysis. ERJ Open Res 2021; 7: 00309-2021 [DOI: 10.1183/23120541.00309-2021].

\section{Abstract}

Background The relationship between asthma and coronavirus disease 2019 (COVID-19) risk is not clear and may be influenced by level of airway obstruction, asthma medication and known COVID-19 risk factors. We aimed to investigate COVID-19 risk in people with asthma.

Methods We used UK Biobank data from all participants tested for severe acute respiratory syndrome coronavirus 2 (SARS-CoV-2) ( $\mathrm{n}=107412 ; 17979$ test positive). Questions at baseline defined ever asthma and asthma medications. Baseline forced expiratory volume in $1 \mathrm{~s}\left(\mathrm{FEV}_{1}\right)$ was categorised into quartiles. Logistic regression modelled relationships between asthma, and asthma categories (age at onset, medications, $\mathrm{FEV}_{1}$ quartiles), and risk of SARS-CoV-2 positive test. We investigated modification by sex, ethnic group, smoking and body mass index.

Results There was a reduced risk of a positive test associated with early-onset asthma ( $<13$ years) (OR 0.91, 95\% CI 0.84-0.99). This was found for participants with early-onset asthma who were male (OR 0.87, 95\% CI 0.78-0.98), nonsmokers (OR 0.87, 95\% CI 0.78-0.98), overweight/obese (OR 0.85, 95\% CI $0.77-0.93$ ) and non-Black (OR 0.90, 95\% CI 0.82-0.98). There was increased risk amongst early-onset individuals with asthma in the highest compared to lowest quartile of lung function (1.44, 1.05-1.72).

Conclusion Amongst male, nonsmoking, overweight/obese and non-Black participants, having early-onset asthma was associated with lower risk of a SARS-CoV-2 positive test. We found no evidence of a protective effect from asthma medication. Individuals with early-onset asthma of normal weight and with better lung function may have lifestyle differences placing them at higher risk. Further research is needed to elucidate the contribution of asthma pathophysiology and different health-related behaviour, across population groups, to the observed risks.

\section{Introduction}

As of July 14, 2021, the severe acute respiratory syndrome coronavirus 2 (SARS-CoV-2) pandemic has infected over 187 million people globally and is responsible for over 4 million deaths (John Hopkins Coronavirus Resource Centre). It is unclear if adults with asthma are particularly susceptible to SARS-CoV-2 infection and/or have a worse prognosis if infected than individuals without asthma. Given that asthma affects $>339$ million people worldwide [1], these are important questions for people with asthma, their families and healthcare providers.

Early studies in China reported an unexpectedly low prevalence of asthma in hospital admitted coronavirus disease 2019 (COVID-19) patients compared to the general population [2]. Yet, subsequent reports provided conflicting results with some studies finding a reduced asthma prevalence in patients with 
COVID-19 [3], and others reporting similar [4, 5] or increased prevalence compared with the local general population [6]. Failure to account for other factors like age, sex, smoking, ethnic group and body mass index (BMI) may contribute to the inconsistent findings [7, 8].

There are several hypotheses to explain the potentially lower risk of COVID-19 in patients with asthma. People with asthma may be practising stricter preventive/protective measures to avoid exposure to SARS-CoV-2, especially those with severe asthma, which is correlated with forced expiratory volume in $1 \mathrm{~s}\left(\mathrm{FEV}_{1}\right)$ [9]. Furthermore, individuals with severe asthma are more likely to be on asthma treatment, especially inhaled (ICS) or oral corticosteroids, which may be protective for SARS-CoV-2 infection [10-12]. Therefore, asthma medication and $\mathrm{FEV}_{1}$ need to be considered in the analysis. Lastly, the $\mathrm{TH}_{2}$ predominant immune response found in patients with asthma may be protective [13].

Addressing the above research gaps may potentially inform decisions around risk and preventive treatment during the pandemic. Therefore, in the UK Biobank cohort tested for SARS-CoV-2, we sought to investigate: 1) the association between asthma and a positive SARS-CoV-2 test; 2) the association between asthma, stratified across age-of-onset phenotypes, $\mathrm{FEV}_{1}$ categories and asthma treatment categories, and a positive SARS-CoV-2 test; and 3) how the asthma-SARS-CoV-2 relationship may be modified by sex, BMI, ethnic group and smoking.

\section{Methods}

Study population: UK Biobank Cohort

Detailed information on the design and methodologies of the UK Biobank Cohort have been described previously (https://www.ukbiobank.ac.uk/). Briefly, it recruited 500000 participants aged 40-69 years living close to one of 22 assessment centres across England, Scotland and Wales, between the years 2007 and 2010. The data on SARS-CoV-2 infection is derived from the entire cohort. At recruitment participants undertook a range of measurements, including questionnaires regarding basic demographic information, lifestyle and disease history, with linkages to electronic medical records and pre-bronchodilator spirometry. Ethnic group and smoking status were self-reported. Participant medical histories were based on self-reported doctor diagnosis, which was verified during the assessment interview.

\section{Ethics approval}

The current analysis was approved by the North West MultiCentre Research Biobank ethics committee (UKB application 28502) and by the Human Research Ethics Committee at the University of Melbourne (Approval ID 2057006.2).

\section{Definitions and data collection \\ Exposures}

We identified participants as having "ever asthma" [14] if they reported asthma in a verbal interview at the time of recruitment to UK Biobank or if they answered "asthma" to the touch-screen question "Has a doctor ever told you that you have had any of the following conditions?” at any of the follow-ups. Age of asthma onset was classified into temporal phenotypes of early onset ( $<13$ years of age) or late onset $(\geqslant 13$ years of age).

A three-level, mutually exclusive variable was generated using asthma medication codes collected during the verbal interview at the UK Biobank Assessment Centre, to categorise medication use for asthma into three groups: 1) no medication, 2) medications other than steroids or 3) steroid medications (including inhaled corticosteroids and oral steroids \pm other medications).

$\mathrm{FEV}_{1}$ derived from spirometry was divided into quartiles of $\mathrm{FEV}_{1}$ z-scores. Spirometry testers were healthcare technicians or nurses certified to conduct the assessments, and all spirometry measures were performed in accordance with American Thoracic Society/European Respiratory Society (ATS/ERS) guidelines [15], using a Vitalograph Pneumotrac 6800 (Vitalograph, Lenexa, KS, USA). The Biobank spirometry protocol has been published elsewhere [16].

\section{Outcome}

Our outcome of interest was SARS-CoV-2 test positive compared to test negative. The test used for SARS-CoV-2 detection was a polymerase chain reaction (PCR) test considered the current gold standard worldwide. UK Biobank data was linked to national SARS-CoV-2 laboratory test data through Public Health England (PHE). Data provided included SARS-CoV-2 test results, specimen origin at the time of testing (hospital inpatient versus other settings). Data used were for the period April 1, 2020 to June 23, 2021 (including specimen data until June 14, 2021). 
TABLE 1 Demographic characteristics amongst all UK Biobank individuals who attended an assessment centre and amongst UK Biobank individuals with severe acute respiratory syndrome coronavirus 2 (SARS-CoV-2) test data

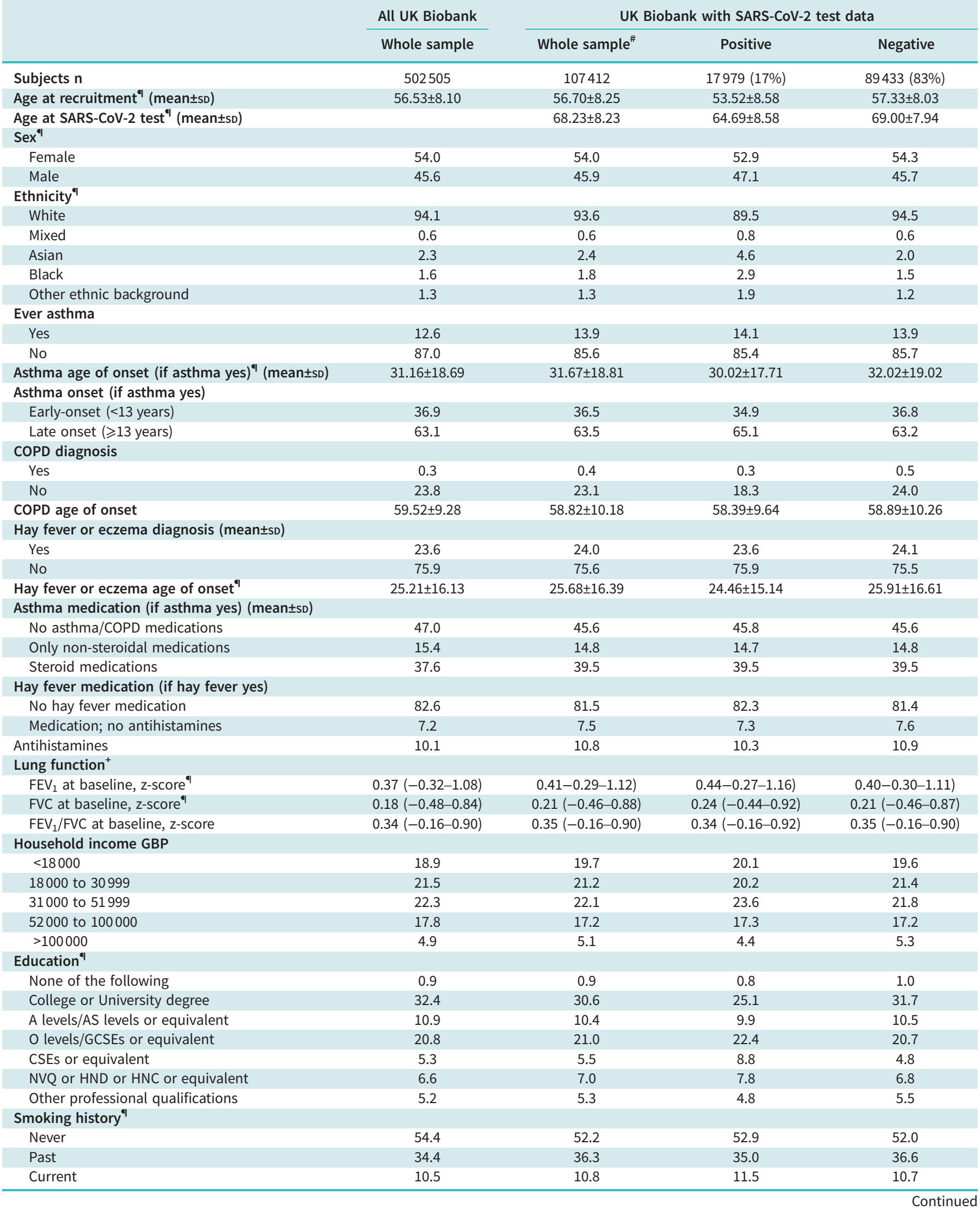


TABLE 1 Continued

\begin{tabular}{|c|c|c|c|c|}
\hline & \multirow{2}{*}{$\begin{array}{l}\text { All UK Biobank } \\
\text { Whole sample }\end{array}$} & \multicolumn{3}{|c|}{ UK Biobank with SARS-CoV-2 test data } \\
\hline & & Whole sample ${ }^{\#}$ & Positive & Negative \\
\hline Smoking pack-years & $19.00(10.00-32.00)$ & $19.88(10.13-33.00)$ & $20.00(10.50-33.00)$ & $19.88(10.00-33.00)$ \\
\hline $\mathrm{BMI}^{\top}($ mean $\pm \mathrm{SD})$ & $27.43 \pm 4.80$ & $27.83 \pm 4.96$ & $28.29 \pm 5.10$ & $27.74 \pm 4.93$ \\
\hline White blood cell count" & $6.65(5.63-7.86)$ & $6.70(5.69-7.90)$ & $6.73(5.70-7.99)$ & $6.70(5.69-7.90)$ \\
\hline Eosinophil count & $0.14(0.10-0.21)$ & $0.14(0.10-0.21)$ & $0.14(0.10-0.22)$ & $0.14(0.10-0.21)$ \\
\hline \multicolumn{5}{|l|}{ Close to major road } \\
\hline Yes & 7.0 & 7.2 & 7.4 & 7.1 \\
\hline No & 91.5 & 91.5 & 91.4 & 91.5 \\
\hline Greenspace percentage, buffer $300 \mathrm{~m}$ & $29.53(17.22-48.41)$ & $29.53(17.17-48.21)$ & $27.44(16.37-44.02)$ & $29.97(17.35-49.11)$ \\
\hline
\end{tabular}

Values are $\%$ of participants, mean \pm SD or median (interquartile range) unless stated otherwise. FEV $\mathrm{V}_{1}$ : forced expiratory volume in $1 \mathrm{~s}$; FVC: forced vital capacity; BMI: body mass index. \#: sample of UK Biobank with SAR-CoV-2 test data differs from whole UK Biobank cohort $(p<0.05)$ in all parameters except greenspace percentage, buffer $300 \mathrm{~m}$. ^: all $p$-values are $<0.001$, except for FVC at baseline, $z$-score $(p=0.002)$ and white blood cell count $(p=0.008)$. $p$-values are comparing positive and negative test result: chi-square for categorical exposures, ANOVA for parametric and Mann-Whitney U-test (Wilcoxon rank sum test) for non-parametric analysis of variance for continuous variables. ${ }^{+}$: z-score means represent the mean standard deviation of lung function parameters compared with values for age-, sex- and race-matched individuals.

\section{Other factors (confounders and effect modifiers) \\ Confounders}

We considered baseline information collected on age, sex, highest level of education, average household income, smoking, BMI, ethnic group, white blood cell count, eosinophil count, proximity to a major road, greenspace percentage, and other allergic and respiratory conditions as confounders (table 1). The minimum set of confounders for adjustment was determined by our knowledge of the field and by using causal modelling theory [17] (Directed Acyclic Graph, figure 1).

\section{Effect modifiers}

Baseline smoking status was categorised as "never smoked", "past smoker" or "current smoker", derived from participant responses to the question "do you smoke tobacco now?" and subsequently "in the past, how often have you smoked tobacco?". BMI was categorised according to height and weight measured during the initial assessment centre visit. Ethnic group was defined from a series of sequential branching questions asked during the initial assessment centre visit as part of the touch-screen questionnaire.

\section{Statistical analysis}

Causal modelling theory was used to model the relationship between asthma and a SARS-CoV-2 positive result. We used Directed Acyclic Graphs (DAG) in order to explore potential confounders and to determine those to include in final models (figure 1).

Results are presented as adjusted odds ratios and corresponding 95\% confidence intervals. Logistic regression analyses were performed to estimate: 1) the association between ever asthma (and age of asthma onset) and a SARS-CoV-2 positive test in the UK Biobank participants for whom test results were available; 2) the association of asthma medication use; and 3) asthma stratified by quartiles of baseline pre-bronchodilator $\mathrm{FEV}_{1}$ with a SARS-CoV-2 positive test. Adjustments were made for confounders listed above. A further model was performed including a limited number of confounders (age at recruitment, sex, smoking history, BMI) to address the possibility that some of the included confounders may be mediators. As no appreciable difference was demonstrated (supplementary Table S2), we continued with the fully adjusted model. These models were applied to the entire sample and further analyses of $\mathrm{FEV}_{1}$ quartiles and medication use were limited to participants with asthma (tables from the asthma subsample are included in the supplement). We also performed interaction analyses for sex, smoking, obesity, and ethnic group and a positive test, and stratified results are presented with interaction terms tested using likelihood ratio tests. Owing to low numbers of observations when stratified, self-reported ethnic group was re-categorised as "Black" or "non-Black", in accordance with current literature indicating "Black" ethnic group as a possible risk factor. Similarly, BMI was classified as "normal weight" or "overweight/obese" and smoking history groups "past and current" were collapsed to one "ever smoked" group. All analyses were performed with Stata 15 (StataCorp, College Station, TX, USA). 


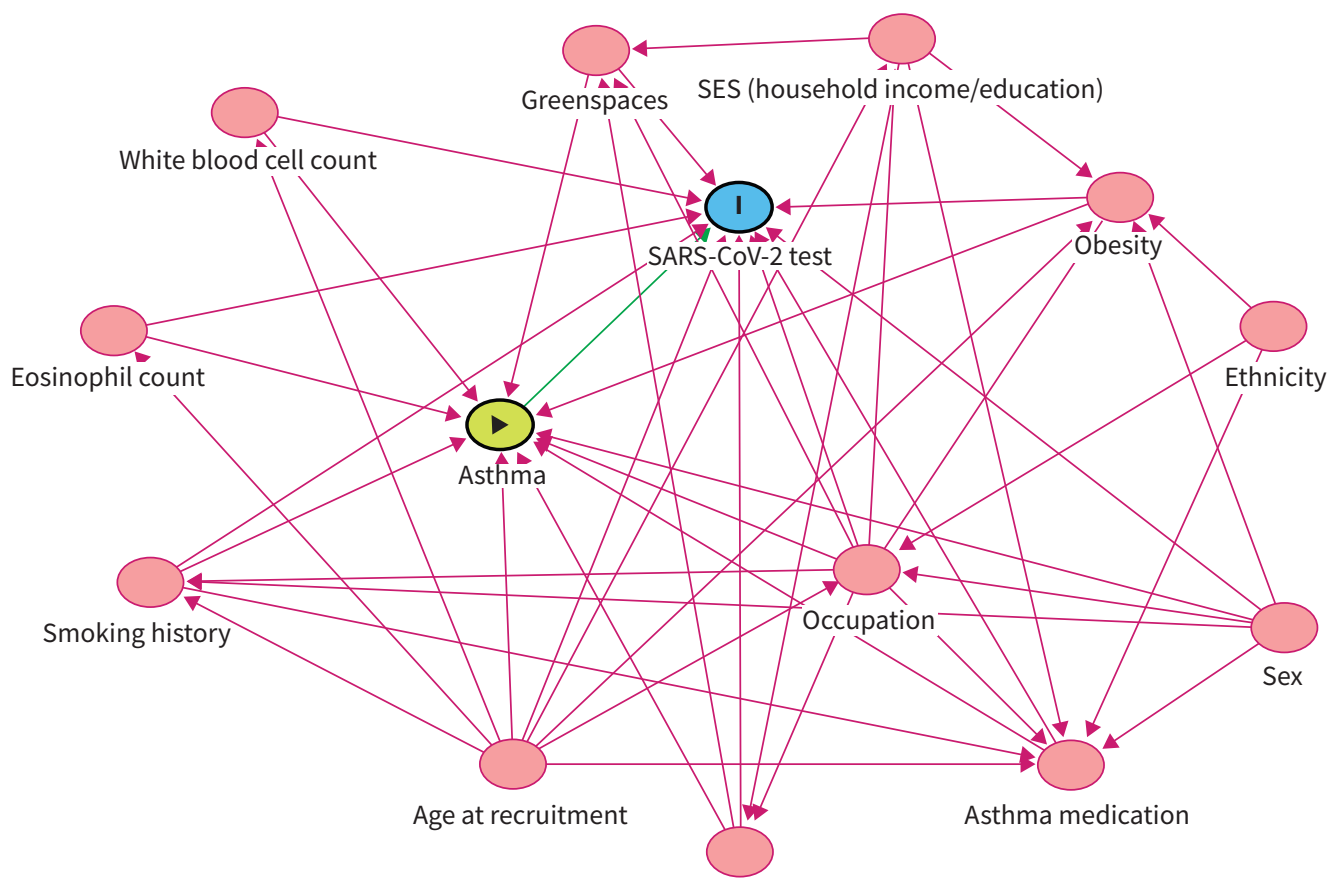

Proximity to major road

FIGURE 1 Directed Acyclic Graph (DAG) identifying potential confounders for the relationship between asthma and severe acute respiratory syndrome coronavirus 2 (SARS-CoV-2) positive test. An arrow from a factor to another means possible association. Red circles indicate variables that require adjustment to assess the relationship between exposure (asthma, green) and the outcome (SARS-CoV-2 test, blue).

Results

Study population

The distribution of demographic characteristics for all UK Biobank participants, and those with SARS-CoV-2 test data, stratified by positive and negative test results is presented in table 1 (supplementary Table S2 contains participant numbers). When the tested sample was compared with the entire English UK Biobank (table 1), there were no or only slight differences in the proportions for most demographic/baseline variables that were statistically significant due to the very large sample size. The percentages differed slightly for the following parameters, where people being tested had: more asthma ever (14\% versus 13\%), less early-onset asthma (36\% versus 37\%), more use of steroid medications (39\% versus $38 \%$ ) and antihistamines (11\% versus $10 \%$ ), better baseline lung function (z-scores: FEV $_{1} 0.41$ versus 0.37 ; forced vital capacity (FVC) 0.21 versus 0.18 ; $\mathrm{FEV}_{1} / \mathrm{FVC} 0.35$ versus 0.34 ), slightly lower levels of the socioeconomic status indicators (income and education) and more total, ever and current smoking.

A total of 107412 UK Biobank participants (mean age 68.23 years) had SARS-CoV-2 test data, of which 17979 (17\%) were positive and 89433 (83\%) negative. The mean \pm sD age of $64.7 \pm 8.6$ years for SARS-CoV-2 test positive participants was lower than for those with a negative test (69.0 \pm 7.9 years) $(\mathrm{p}<0.001)$. The whole cohort and the sample used in this analysis was predominately of "white" ethnicity (both 94\%); however, other ethnic groups demonstrated a relatively higher proportion of positive test results. The percentage of reported doctor-diagnosed asthma at baseline was the same amongst the positive and negative test groups (14\%; $\mathrm{p}=0.458)$. Similarly, the percentage of asthma medication used (both steroid and other) did not differ between participants with or without a positive test.

\section{Association between asthma and a SARS-CoV-2 positive test}

For all tested participants ( $\mathrm{n}=107412)$, the risk of a SARS-CoV-2 positive test was non-significantly lower in those with ever asthma versus those without ever asthma (3\% reduction in odds, 95\% CI 0.92-1.02) (table 2). On phenotyping into individuals with early and late-onset asthma, there was a reduced risk of a SARS-CoV-2 positive test in the early-onset group (9\% reduction in odds, 95\% CI 0.84-0.99) but not in the late-onset group compared to non-asthma individuals. We found no association between asthma 
treatment groups or different quartiles of lung function in all participants with asthma and risk of test positivity when compared with participants without asthma (table 2). When stratified by onset phenotypes (table 3), there was an increased risk for individuals with early-onset asthma with the highest lung function compared with the lowest quartile (44\% increased odds, 95\% CI 1.05-1.72). There was also some evidence for an increased risk in individuals with early-onset asthma treated with steroids but confidence intervals crossed 1 (18\% increase, 95\% CI 0.98-1.42).

The cohort tested for SARS-CoV-2 while in hospital (Origin=1), were more likely to test positive if they had ever had asthma (OR 1.10, 95\% CI 0.99-1.23), and if they were using steroids (OR 1.32, 95\% CI 1.14-1.54), while these associations were not seen for non-hospital samples (supplementary Table S3).

When the analysis was restricted to those with asthma, again, there was no evidence of associations for asthma treatment or lung function and risk of positive SARS-CoV-2 test (supplementary Table S4).

Sex, smoking, ethnicity and BMI by asthma-onset phenotypes (table 4)

The association between early-onset asthma and a SARS-CoV-2 positive test differed by sex (table 4). Males with early-onset asthma had $14 \%$ reduction in the odds of a positive test (95\% CI $0.76-0.97$ ) compared to males without asthma. This was not the case for female participants ( $\mathrm{P}_{\text {int }}$ early-onset asthma/ sex 0.29).

Associations also differed by smoking status. Nonsmokers with early-onset asthma had $13 \%$ reduced odds of a positive test compared with non-asthmatic nonsmokers (95\% CI 0.78-0.98) ( $\mathrm{P}_{\text {int }}$ early-onset asthma/ smoking 0.45).

Amongst participants who were overweight/obese, those with early-onset asthma had $15 \%$ reduced odds of testing positive for SARS-CoV-2 (95\% CI 0.77-0.93) ( $\mathrm{P}_{\text {int }}$ early-onset asthma/BMI 0.01).

Amongst participants with self-reported "non-Black" ethnicity, those with early-onset asthma had 10\% decreased odds of testing positive to SARS-CoV-2 (95\% CI 0.82-0.98) compared to "non-Black" participants without asthma. Conversely, amongst participants with self-reported "Black" ethnicity, those with asthma had 1.5 times increased odds of a positive SARS-CoV-2 test, but 95\% confidence intervals included the null value (0.90-2.6) ( $\mathrm{P}_{\text {int }}$ early-onset asthma/ethnicity 0.14$)$.

Sample sizes were too small to stratify by ethnic group for the relationship between treatment in individuals with asthma and SARS-CoV-2 test status.

\section{Discussion}

Using UK Biobank data from 107412 participants who had been tested for SARS-CoV-2, we observed evidence for a lower risk of a SARS-CoV-2 positive test in those with early-onset asthma versus those without asthma. This association was limited to males, nonsmokers, overweight/obese and participants of non-Black ethnicity. Paradoxically, we also found that individuals with early-onset asthma in the highest quartile of lung function were at greater risk compared to those in the lowest quartile.

\begin{tabular}{|c|c|c|c|}
\hline & Percentage of positive tests $(\mathrm{n} / \mathrm{N})$ & OR $(95 \% \mathrm{Cl})$ for a positive test & $\mathrm{p}$-value \\
\hline \multicolumn{4}{|l|}{ Ever asthma } \\
\hline No & $16.6(15347 / 91977)$ & Ref & \\
\hline Yes & $16.9(2539 / 14988)$ & $0.97(0.92-1.02)$ & 0.28 \\
\hline \multicolumn{4}{|l|}{ Asthma onset } \\
\hline No asthma & $16.6(15347 / 91977)$ & Ref & \\
\hline Early-onset ( $<13$ years) & $16.2(887 / 5469)$ & $0.91(0.84-0.99)$ & 0.03 \\
\hline Late onset ( $\geqslant 13$ years) & $17.3(1652 / 9519)$ & $1.00(0.94-1.07)$ & 0.87 \\
\hline
\end{tabular}


TABLE 3 The associations between asthma age-of-onset phenotypes and severe acute respiratory syndrome coronavirus 2 (SARS-CoV-2) positive test by treatment and lung function

\begin{tabular}{cccccc}
\multicolumn{2}{c}{ Early onset } & \multicolumn{2}{c}{ Late onset } \\
$\begin{array}{cccc}\text { Percentage of positive tests } \\
(\mathrm{n} / \mathrm{N})\end{array}$ & $\begin{array}{c}\text { OR }(95 \% \mathrm{Cl}) \text { for a } \\
\text { positive test }\end{array}$ & & $\begin{array}{c}\text { Percentage of positive tests } \\
(\mathrm{n} / \mathrm{N})\end{array}$ & $\begin{array}{c}\text { OR }(95 \% \mathrm{Cl}) \text { for a positive } \\
\text { test }\end{array}$
\end{tabular}

\begin{tabular}{|c|c|c|c|c|}
\hline \multicolumn{5}{|l|}{ Asthma treatment } \\
\hline No & $15.8(491 / 3114)$ & Ref & $18(672 / 3727)$ & Ref \\
\hline $\begin{array}{l}\text { Treated with } \\
\text { non-steroid }\end{array}$ & $17.3(112 / 645)$ & $1.05(0.81-1.36), p=0.67$ & $16.5(261 / 1576)$ & $0.90(0.75-1.08), p=0.26$ \\
\hline Treated with steroids & $16.6(284 / 1710)$ & $1.18(0.98-1.42), p=0.07$ & $17.1(719 / 4216)$ & $1.04(0.91-1.19), p=0.55$ \\
\hline \multicolumn{5}{|l|}{$\begin{array}{l}\text { Quartiles of baseline } \\
\text { FEV }_{1}\end{array}$} \\
\hline $\begin{array}{l}\text { Q1 of FEV } \text { (lowest } \\
\text { quartile) }\end{array}$ & $12.4(70 / 562)$ & Ref & $18.3(190 / 1035)$ & Ref \\
\hline Q2 of FEV 1 & $16.7(120 / 715)$ & $1.25(0.88-1.79), p=0.20$ & $15.2(203 / 1332)$ & $0.81(0.64-1.03), p=0.09$ \\
\hline Q3 of $\mathrm{FEV}_{1}$ & $14.8(145 / 976)$ & $1.22(0.87-1.72), p=0.23$ & $16.9(280 / 1653)$ & $0.92(0.73-1.15), p=0.4 \varepsilon$ \\
\hline Q4 of $\mathrm{FEV}_{1}$ & $16.7(310 / 1851)$ & $1.44(1.05-1.72), p=0.02$ & $17.4(516 / 2962)$ & $0.90(0.73-1.15), p=0.36$ \\
\hline
\end{tabular}

\section{Previous studies}

ATKINs et al. [6] studied 507 participants from UK Biobank and found that asthma was not a protective or a risk factor for a SARS-CoV-2 positive test in fully adjusted models. The smaller number of patients with asthma included in that study may have limited power to observe associations as compared to the present study (90 versus 14988). In keeping with our study, they found evidence for interaction by sex, so amongst patients with asthma, females were at greater risk of COVID-19 hospitalisation (but not death) [6]. On the other hand, WILLIAMSON et al. [7], using the OpenSAFELY platform, comprising data from the NHS of over 17 million people in England, analysed factors related to 10926 COVID-19 deaths and found evidence of increased risk of death for male sex, older age, severe asthma, ethnicity (Black and South Asian), smoking and obesity (BMI $>40$ ). Severe asthma in this study was defined by prescription of oral corticosteroid in the past 12 months. In our analyses, quartiles of lung function served as a proxy for asthma severity, and we found some contrasting evidence that participants with early-onset asthma in the highest quartile of lung function were at greater risk of SARS-CoV-2 positivity than people without asthma.

\section{Interpretation of findings}

We believe that our findings are underpinned by a mix of biological and behavioural mechanisms. Several mechanisms may explain our observation that asthma may be protective for COVID-19 in the early-onset asthmatic participants. Firstly, as we have limited our analysis to the sample in the UK Biobank tested for SARS-CoV-2, there may be a degree of collider bias [18] since patients with asthma may have different health-related behaviour and undertake more stringent protective and avoidance measures as well as seeking healthcare earlier for potential COVID-19 symptoms, so there may be reduced positive test results in this group. However, the sample tested is very large (107000) and the prevalence of asthma in the tested cohort was very similar to the entire UK Biobank cohort (14\% versus 13\%), and the prevalence of positive tests amongst those with asthma was the same (17\%) as the entire tested sample.

Secondly, the immunological characteristics of patients with asthma may be protective for SARS-CoV-2 infection. Asthma is characterised by increased populations of both $\mathrm{CD}^{+}$and $\mathrm{CD}^{+}$cells and reduced expression of the SARS-CoV-2 receptor, angiotensin-converting enzyme 2 (ACE2) [19]. There is evidence that reduced levels of $\mathrm{CD}^{+}$and $\mathrm{CD}^{+}$cells are associated with increased risk of COVID-19 disease and increased severity [13], so greater levels in people with asthma may be protective. Notably, there are two major forms of asthma characterised by specific immune responses. Atopic asthma is characterised by a TH2 response and increased levels of interleukin (IL)-4, IL-5, IL-9 and IL-13 cytokines. Conversely, non-atopic asthma is characterised by an increased TH1 response with increased levels of interferon- $\gamma$ (IFN- $\gamma$ ) and IL-2, IL-12 and IL-17 cytokines. There is some evidence to suggest that atopic asthma with a TH2 response may be more protective through downregulation of ACE2 cell surface receptors. The SARS-CoV-2 virus is known to enter host cells via the ACE2 receptor using transmembrane protease serine 2 (TMPRSS2) [20]. Analysis of the URECA cohort found that increasing atopy was associated with 


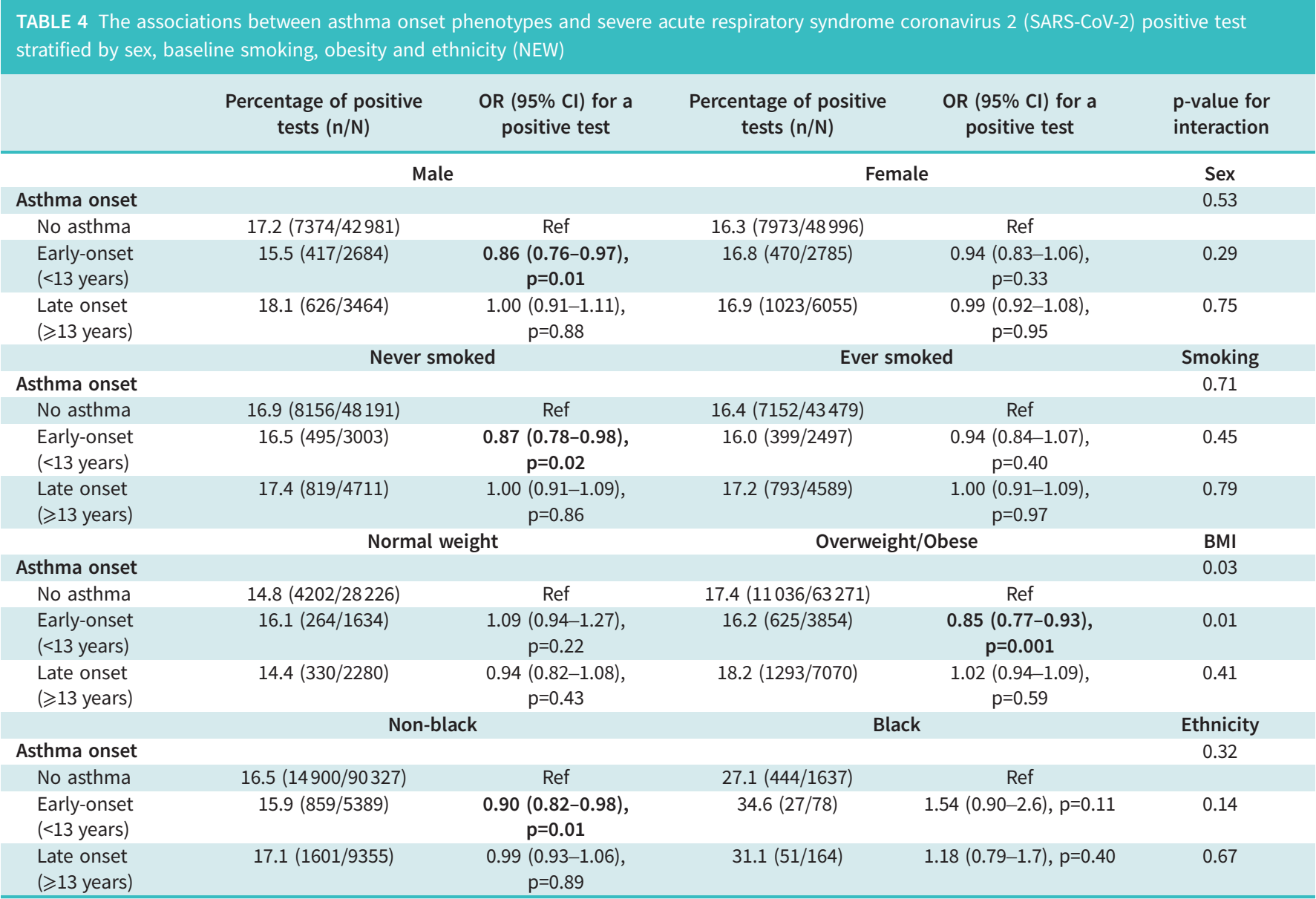

Adjusted for age at recruitment, sex, and baseline measures of: education, average household income, smoking history, BMI, ethnicity, white blood cell count, eosinophil count, close to major road and greenspaces buffer. Bold values indicate $p$-value $<0.05$.

reduced ACE2 expression in nasal epithelium when compared to children with asthma and no atopy [19]. Conversely, non-atopic asthma was not associated with reduced ACE2 expression [19]. Therefore, atopic asthma may be protective through reduced ACE2 expression on cell surfaces limiting viral entry. This hypothesis has received further support from a recent publication finding that amongst hospital inpatients with COVID-19 and asthma, those with an allergic phenotype (history, eosinophils, cytokines) had less severe COVID-19 disease [21]. The prevalence of non-atopic asthma in adults is around $50 \%$ and is far more frequent in women [22]. In our analyses, we found evidence that sex differences may be associated with risk amongst people with asthma. Men, who are more likely to have early-onset atopic asthma [23], were protected compared with women. This may be partly explained by the adult atopic/non-atopic asthma phenotypes. This sex difference for COVID-19 risk amongst adults with asthma has now been found in several studies $[5,24,25]$.

Thirdly, asthma treatments may be protective for SARS-CoV-2 infection. There is evidence that both steroid [26] and non-steroid medications [10] may modify cellular entry of SARS-CoV-2 through downregulation of cell surface ACE2 expression and interference with the action of TMPRSS2, respectively. In this way, asthma medications may reduce SARS-CoV-2 cellular entry and protect against both SARS-CoV-2 infection and severity. Owing to an apparent dose-response relationship between increased ICS and reduced prevalence of cell surface ACE2 receptors [26], there has been a call for randomised controlled trials $[27,28]$ and several are underway (NCT04331054, NCT04330586). SCHULTZE et al. [29] used the OpenSAFELY platform to investigate the association between ICS use and COVID-19-related death. From 820000 people with asthma, they found evidence that those taking higher doses of ICS had a higher risk of death than those on medium to low doses. Further modelling suggested that these differences may be due to underlying confounding related to the overall health of the individual and associated comorbidities. They concluded that there was no major protective role from ICS for 
COVID-19. In line with this, we also did not observe a protective effect of asthma medication on risk of a positive SARS-CoV-2 test.

Finally, we also found evidence amongst overweight/obese individuals that early-onset asthma may be associated with protection, and better lung function may be associated with increased risk for SARS-CoV-2 infection. These are counterintuitive findings, and we postulate that the mechanism may be from health-related behaviours. People with asthma who are overweight/obese may be less active in the community and more inclined to follow COVID-19 health directives, limiting their exposure to SARS-CoV-2. In contrast, individuals with early-onset asthma with good lung function and likely mild asthma may be more active and less likely to observe all COVID-19 precautionary measures.

\section{Strengths and limitations}

Major strengths of this study are its large sample size, that asthma disease status was ascertained prior to the COVID-19 pandemic, along with potential confounders and effect modifiers, and that we had prior objective measurements of lung function that allowed us to classify asthma objectively into proxy severity groups. Potential limitations include that the group is limited to older adults, and that the sample tested, in contrast to the entire UK Biobank cohort, may differ in health-related behaviours that may also be related to asthma and asthma severity. Ascertainment of doctor-diagnosed asthma was from questionnaire, and although this method has been found to have high sensitivity/specificity [30], it may have led to a degree of non-differential misclassification. Given that, associations found may be underestimates of true associations. Furthermore, participants of the UK Biobank are known to be a healthier, more educated group than the general population. Despite this, our findings show considerable overlap with those from the large population-based NHS studies [7, 29].

\section{Conclusions}

From UK Biobank data, there is evidence that early-onset asthma is protective for SARS-CoV-2 infection in specific groups: male, nonsmoking, overweight/obese and non-Black participants. Lack of asthma phenotyping in previous research may have led to inconsistent associations. Further research is required to investigate the impact of health-related behaviour in people with asthma and likely mechanisms before a causal association can be implied.

Acknowledgements: This research has been conducted using the UK Biobank Resource and we thank all participants for their generous contribution to this outstanding resource (UK Biobank Project ID: 28502).

Author contributions: All authors involved in conception, analysis and/or interpretation of the findings. C.J. Lodge and A. Doherty wrote the initial draft to which all authors contributed.

Provenance: Submitted article, peer reviewed.

Conflict of Interest: C.J. Lodge, D.S. Bui, R. Cassim, A.J. Lowe and S.C. Dharmage are investigators on a GlaxoSmithKline grant that is unrelated to and had no influence on this work. A. Doherty has nothing to disclose. A.J. Lowe reports receiving Research Fellowships from National Health and Medical Research Council, during the conduct of the study. A. Agusti has nothing to disclose. M.A. Russell has nothing to disclose.

Support statement: This work was funded by a grant from the University of Melbourne.

References

1 Vos T, Abajobir AA, Abate $\mathrm{KH}$, et al. Global, regional, and national incidence, prevalence, and years lived with disability for 328 diseases and injuries for 195 countries, 1990-2016: a systematic analysis for the Global Burden of Disease Study 2016. Lancet 2017; 390: 1211-1259.

2 Li X, Xu S, Yu M, et al. Risk factors for severity and mortality in adult COVID-19 inpatients in Wuhan. $J$ Allergy Clin Immunol 2020; 146: 110-118.

3 Garcia-Pachon E, Zamora-Molina L, Soler-Sempere MJ, et al. Asthma prevalence in patients with SARS-CoV-2 infection detected by RT-PCR not requiring hospitalization. Respir Med 2020; 171: 106084.

4 Butler MW, O'Reilly A, Dunican EM, et al. Prevalence of comorbid asthma in COVID-19 patients. J Allergy Clin Immunol 2020; 146: 334-335.

5 Chhiba KD, Patel GB, Vu THT, et al. Prevalence and characterization of asthma in hospitalized and non-hospitalized patients with COVID-19. J Allergy Clin Immunol 2020; 146: 307-314.

6 Atkins JL, Masoli JAH, Delgado J, et al. Preexisting comorbidities predicting COVID-19 and mortality in the UK Biobank community cohort. J Gerontol A Biol Sci Med Sci 2020; 75: 2224-2230. 
7 Williamson EJ, Walker AJ, Bhaskaran K, et al. Factors associated with COVID-19-related death using OpenSafely. Nature 2020; 584: 430-436.

8 Morais-Almeida M, Pité H, Aguiar R, et al. Asthma and the coronavirus disease 2019 pandemic: a literature review. Int Arch Allergy Immunol 2020; 181: 680-688.

9 Firoozi F, Lemière C, Beauchesne MF, et al. Development and validation of database indexes of asthma severity and control. Thorax 2007; 62: 581-587.

10 Cashman DP. Why the lower reported prevalence of asthma in patients diagnosed with COVID-19 validates repurposing EDTA solutions to prevent and manage treat COVID-19 disease. Med Hypotheses 2020; 144: 110027.

11 Halpin DMG, Singh D, Hadfield RM. Inhaled corticosteroids and COVID-19: a systematic review and clinical perspective. Eur Respir J 2020; 55: 2001009.

12 Horby P, Lim WS, Emberson J, et al. Dexamethasone in hospitalized patients with COVID-19: preliminary report. N Engl J Med 2021; 384: 693-704.

13 Poland GA, Ovsyannikova IG, Kennedy RB. SARS-CoV-2 immunity: review and applications to phase 3 vaccine candidates. Lancet 2020; 396: 1595-1606.

14 Allen NE, Sudlow C, Peakman T, et al. UK Biobank Data: Come and get it. Sci Transl Med 2014; 6: 224ed224.

15 Miller MR, Hankinson J, Brusasco V, et al. Standardisation of spirometry. Eur Respir J 2005; 26: 319-338.

16 De Matteis S, Jarvis D, Hutchings S, et al. Occupations associated with COPD risk in the large population-based UK Biobank cohort study. Occup Environ Med 2016; 73: 378-384.

17 VanderWeele TJ, Robins JM. Directed acyclic graphs, sufficient causes, and the properties of conditioning on a common effect. Am J Epidemiol 2007; 166: 1096-1104.

18 Griffith GJ, Morris TT, Tudball MJ, et al. Collider bias undermines our understanding of COVID-19 disease risk and severity. Nat Commun 2020; 11: 5749.

19 Jackson DJ, Busse WW, Bacharier LB, et al. Association of respiratory allergy, asthma, and expression of the SARS-CoV-2 receptor ACE2. J Allergy Clin Immunol 2020; 146: 203-206.e203.

20 Hoffmann M, Kleine-Weber H, Schroeder S, et al. SARS-CoV-2 cell entry depends on ACE2 and TMPRSS2 and is blocked by a clinically proven protease inhibitor. Cell 2020; 181: 271-280.e278.

21 Muñoz X, Pilia F, Ojanguren I, et al. Is asthma a risk factor for COVID-19? Are phenotypes important? ERJ Open Research 2021; 7: 00216-2020.

22 Leynaert B, Sunyer J, Garcia-Esteban R, et al. Gender differences in prevalence, diagnosis and incidence of allergic and non-allergic asthma: a population-based cohort. Thorax 2012; 67: 625-631.

23 Shah R, Newcomb DC. Sex bias in asthma prevalence and pathogenesis. Front Immunol 2018; 9: 2997.

24 Beurnier A, Jutant EM, Jevnikar M, et al. Characteristics and outcomes of asthmatic patients with COVID-19 pneumonia who require hospitalisation. Eur Respir J 2020; 56: 2001875.

25 Lovinsky-Desir S, Deshpande DR, De A, et al. Asthma among hospitalized patients with COVID-19 and related outcomes. J Allergy Clin Immunol 2020; 146: 1027-1034.ed1024.

26 Peters MC, Sajuthi S, Deford P, et al. COVID-19-related genes in sputum cells in asthma. Relationship to demographic features and corticosteroids. Am J Respir Crit Care Med 2020; 202: 83-90.

27 Miyazawa D, Kaneko G. Clinical trials of inhaled beclomethasone and mometasone for COVID-19 should be conducted. J Med Virol 2020; 93: 637-638.

28 Nicolau DV, Bafadhel M. Inhaled corticosteroids in virus pandemics: a treatment for COVID-19? Lancet Respir Med 2020; 8: 846-847.

29 The OpenSAFELY Collaborative, Schultze A, Walker AJ, et al. Inhaled corticosteroid use and risk COVID-19 related death among 966461 patients with COPD or asthma: an OpenSAFELY analysis. medRxiv 2020; preprint [https://doi.org/10.1101/2020.06.19.20135491].

30 Jenkins Mark A, Clarke Jane R, Carlin John B, et al. Validation of questionnaire and bronchial hyperresponsiveness against respiratory physician assessment in the diagnosis of asthma. Int $J$ Epidemiol 1996; 25: 609-616. 